\title{
Analysis of the Relationship between Folk Art Style and Animation Image Design
}

\author{
Liu Tao ${ }^{1, *}$, Xu Xiaoting ${ }^{2}$ \\ ${ }^{1}$ Department of Fine Arts and Design, Dongchang College, Liaocheng University, Shandong, China, \\ 252000 \\ ${ }^{2}$ School of Tourism Management, Liaocheng Vocational and Technical College, Shandong, China, \\ 252000
}

Keywords: Folk art modeling; cartoon image; relationship

\begin{abstract}
The folk art style contains rich traditional cultural knowledge, which not only has a strong symbolic meaning, but also has the role of cultural inheritance. Its connotation always runs through the ideological subconscious of every generation of Chinese people, affecting people's thinking and creation. It is precisely because the local cultural characteristics of folk art styling are obvious that many anime characters have an inseparable relationship with them. In the process of designing anime image, some folk art styling features are borrowed. Therefore, folk art styling is important for the design of avatar images.
\end{abstract}

\section{Current status and main features of folk art styling}

\subsection{The status quo of folk art modeling}

After a long period of accumulation, there are many types of folk art styling, such as woodcut prints, stone wood and bamboo carvings, which all reflect the local folk customs, have a beautiful meaning, and the local colors are very strong, gradually forming a unique artistic style. Folk art is an invaluable artistic heritage, but under the impact of social development, people are increasingly ignoring folk art, and folk art is dying out without protection. In recent years, people have begun to realize the importance of folk art, and draw on the creative thinking and techniques of folk art to design a unique cartoon image. Therefore, relevant people have established a series of protective measures and systems to promote the development of folk art ${ }^{[1]}$.

\subsection{Main features of folk art modeling}

First of all, the folk art style has a flexible and aesthetic perspective. The folk art style has a variety of characteristics and forms a complicated system. This is mainly because after a long period of historical changes, there are great differences in regions and customs, and the unequal levels of creators make the folk art more Variety. In the process of aesthetics of folk art styling, there is a polarized aesthetic state, such as juvenile and mature, and such as rough and delicate, such an aesthetic phenomenon is contradictory to each other, and at the same time is a whole of 
interdependence.

Secondly, the creation of folk art styles follows a certain pattern of image. For thousands of years, folk art has been used as a daily play for children, and some ordinary people use it as a living ornament. There are various forms of artistic expression, and every artistic expression, no matter in terms of creative techniques, In the aesthetic sense, they all have the characteristics of local culture. For example, from the analysis of the usefulness and aesthetics of folk art, the whole is full of perfection. This mainly refers to perfection and completeness. In order to make the overall coordination of the form, it has produced "seeking for perfection, seeking beauty, seeking for the big, and seeking survival." And so on. All in all, from the overall point of view, folk art creation shows a variety of forms of symbolic, general, random and abstract, and can be used to learn this way of thinking when designing anime ${ }^{[2]}$. The texture of folk art styling is shown in Figure 1.

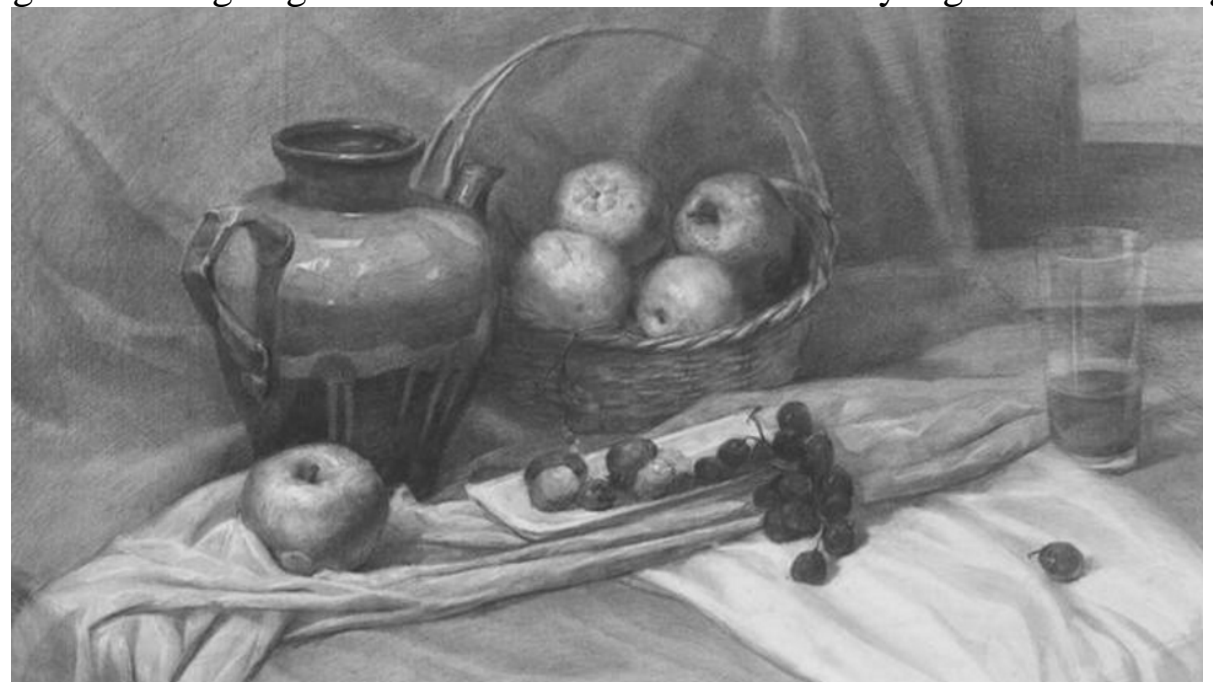

Figure 1 Texture of folk art styling

Finally, the folk art style has a unique creative method. Folk art creators each have different creative thinking, creative methods and aesthetic interest, thus forming a unique plastic art system. Generally speaking, the creative method of folk art modeling is mainly scatter perspective, which mainly refers to the overall form of art from multiple angles and multiple perspectives. From the perspective of various forms of folk art styling, there are mainly the following types of modeling methods: the first modeling method is the theme image expansion method, which mainly refers to the development of things around a subject, and other Modeling as an aid. The second method of modeling is to go deep into the internal method of the object, such as the shaping of the internal texture of the shadow is to use this method. The third modeling method is the non-stationary image method, which mainly refers to the various forms of things that are displayed during the design process. The fourth modeling method is the space-time synthesis method, which mainly refers to the representation of things in different states of time and space. The fifth modeling method is the conformal modeling method, such as the shaping of the butterfly mother. The various styling methods of folk art provide a creative method for the design of anime image. At present, many animation images have been designed and used, and have achieved good results ${ }^{[3]}$.

\subsection{The relationship between folk art and animation}

First of all, there is a certain internal connection between folk art and animation. In some respects, animation is developed from folk art, but folk art is static, and animation is dynamic. Both need to first define a theme and then create around the theme. In addition, the pursuit of folk art 
styling in the process of design is "live and vivid", and this is the same as the design idea of animation, so there is a certain internal relationship between the two. The main characteristics of folk art modeling play an important guiding role in the design of animation characters. These characteristics reflect the expression of the Chinese national connotation, but also cover a variety of design thinking. The design of anime image cannot be completely separated from people's life, but an art form that excavates from life and is higher than life. Based on their experience, knowledge and life experience, the creators use the exquisite technology and exaggeration to express the animation image, give the cartoon characters a distinct character, and embody the life and wishes of the people through fantasy and imagination. come out. Therefore, the design of the animation image is inseparable from the folk art style. By unifying the two together, an animation image with local characteristics will be produced.

\section{Enlightenment of folk art model on cartoon image design}

\subsection{The current status of China's current animation image}

The design of animation images in China is not mature enough. Not only is the image expression not mature enough, but the image creativity is not mature enough. On the whole, it is still in the stage of exploration. It can be seen from the numerous anime images in our country that there are not many original works, and there are fewer unique features. The meaning of the creative imagination is not prominent, slightly monotonous, lacking certain vitality, and not artistic. When imitating, the imitation of Japanese and American animation styles is obvious. The animation with local characteristics is not without, but relatively less. This is mainly because the designers do not understand the traditional culture and cannot use them skillfully ${ }^{[4]}$.

\subsection{The design of different shapes of anime images}

About the design of the animation plane shape. In the folk art flat shape, if different materials are used, the texture effect will be different. For example, from the perspective of dyeing, weaving, clothing, clothing, etc., we have applied many creative techniques such as weaving, dyeing, embroidering, and plastics. The design of the graphic image of the anime image is mainly drawn directly on paper, or directly on the film. This design method can make the animation image have a strong artistic style, and the carving details are also processed. Better, it can make it more lifelike and vivid. For example, through the combination of paper-cutting and shadow-shadow, a flat image space is formed. The image is externally vivid and concise, which can trigger the viewer to fully exert his imagination. The image has inherent fuzzy features, and the viewer can have more Imagine the space and supplement the artistic image in my mind. From the point of view of performance skills, the silhouette shape has a strong contour feature. The outline shape usually does not need to be described. It is necessary to pay attention to the combination of dynamic and modality. This is the key link for the success of silhouette modeling. The weight of influence of art works is shown in Table 1.

Table 1 Weight of influence of art

\begin{tabular}{ccccc}
\hline Index & Color & Pattern & Texture & Material \\
\hline Weight & 0.382 & 0.403 & 0.112 & 0.103 \\
\hline
\end{tabular}

For example, paper-cut anime, as long as the image of the object is focused on the design process, it shows a sense of movement and layering. Of course, the design of the graphic image of the anime image is not only the painting method mentioned above, but also the method of single-line flat 
coating. This method can make the animation image have a unique charm effect. In the specific application process, the designer needs to apply the corresponding painting method according to the actual needs.

The design of the three-dimensional shape of the anime image. The three-dimensional shape of folk art has a variety of expressions. For example, a variety of mud toys designed for the needs of entertainment or folk etiquette, these toys are expressive and close to life. There are also some designs of three-dimensional art stereoscopic styles. Generally, there are mainly four kinds of art styles: the first one is a puppet, the second is a puppet, the third is a puppet, and the fourth is a wire. puppet. The three-dimensional shape of the anime image is essentially a three-dimensional image of the space. This three-dimensional shape has a model joint couple and a pinch couple. In general, the design of the model joint couple mainly focuses on the active joints. The materials used include not only wood, mud and plaster, but also metal joints and skeletons. The fabrication of the couple is mainly a kind of carving and shooting at the same time. Even anime is not only not very risky, but also very contingent. Of course, there is another form of fabrication. This new style is mainly to shape a number of models first, and then replace them when they are officially shot, which is more reliable. These have provided conditions for the development of animation art, which has resulted in a series of animated characters. For example, when the cartoon "simple homework" is designed for the task image, it draws on the design method of the puppet to make the task more vivid.

About the design of computer-generated animation images. The application of computer technology in animation and image design is more and more extensive, resulting in a series of online games, computer animations and so on. The animation image produced by computer technology is mainly a visual illusion generated by using digital technology. There is no need to actually operate things, and no material materials are used. Because it is very different from the above-mentioned plane and three-dimensional modeling, the computer-generated animation image is essentially a non-material shape, but it is not all out of the folk art style, using computer technology for animation. When designing the image, I still refer to some folk art styles. Through computer technology, various materials can be simulated to form various visual effects, thus forming a colorful anime image. After combining the folk art shapes and animations such as puppets, many creative methods are derived. However, regardless of the technology used, Different materials will affect the animation, so designers need to fully consider the particularity of the animation image design. At the same time, when designing the anime image, it also fully utilized the expression techniques such as meticulous brush and ink painting, which was a good combination of scatter composition and freehand technique, so that the whole cartoon has strong local characteristics and achieved good viewing results. , won the grand prize of the Japanese TBS animation digital competition ${ }^{[5]}$.

\section{Conclusion}

Anime image can only have broad development prospects if it reflects the local cultural characteristics. In order to make the animation image have its own characteristics, it is necessary to fully understand the folk art style, on the one hand, master the main characteristics of folk art modeling; on the other hand, master the classification of folk art style, only in this way can be flexible when designing the animation image. Use various types of creative techniques. At the same time, we must hold an open concept, pay attention to the development of modern animation image production technology, apply advanced technology, blend traditional and modern cultural concepts, and design works with profound cultural connotations to form a unique national anime image. 


\section{Acknowledgement}

Shandong social sciences planning research project: analysis and countermeasure research on the current situation of non-standard accommodation in Shandong province from the perspective of regional tourism (no. $16 \mathrm{cglj} 42$ )

\section{References}

[1]Kramskaia N. V.,Kronevald E. V.. Promotion of healthy food by means of design [J]. Interaktivnaâ Nauka, 2018(1 (23)).

[2]Thakur, V.S.,Thakur, K.. Design and Implementation of a Highly Efficient Gray Image Compression Codec Using Fuzzy Based Soft Hybrid JPEG Standard [P]. , 2014.

[3]Boukhayma, A.,Peizerat, A.,Dupret, A.,Enz, C.. Design optimization for low light CMOS image sensors readout chain [P]. New Circuits and Systems Conference (NEWCAS), 2014 IEEE 12th International, 2014.

[4]Mahanta, K.,Das, D.J.,Bhuyan, H.M.K.R.,Dutta, A.,Gogoi, M.. Design and implementation of an MSI number based image watermarking architecture in transform domain [P]. Signal Processing and Integrated Networks (SPIN), 2014 International Conference on, 2014.

[5]Said, Y.,Saidani, T.,Atri, M.. High-level design for image processing on FPGA using Xilinx AccelDSP[P]. Computer Applications and Information Systems (WCCAIS), 2014 World Congress on, 2014. 